\title{
Existence Results for a System of Coupled Hybrid Fractional Differential Equations
}

\author{
Bashir Ahmad, ${ }^{1}$ Sotiris K. Ntouyas, ${ }^{1,2}$ and Ahmed Alsaedi ${ }^{1}$ \\ ${ }^{1}$ Nonlinear Analysis and Applied Mathematics (NAAM) Research Group, Department of Mathematics, Faculty of Science, \\ King Abdulaziz University, P.O. Box 80203, Jeddah 21589, Saudi Arabia \\ ${ }^{2}$ Department of Mathematics, University of Ioannina, 45110 Ioannina, Greece
}

Correspondence should be addressed to Bashir Ahmad; bashirahmad_qau@yahoo.com

Received 22 June 2014; Revised 2 August 2014; Accepted 4 August 2014; Published 18 August 2014

Academic Editor: Praveen Agarwal

Copyright (C) 2014 Bashir Ahmad et al. This is an open access article distributed under the Creative Commons Attribution License, which permits unrestricted use, distribution, and reproduction in any medium, provided the original work is properly cited.

\begin{abstract}
This paper studies the existence of solutions for a system of coupled hybrid fractional differential equations with Dirichlet boundary conditions. We make use of the standard tools of the fixed point theory to establish the main results. The existence and uniqueness result is elaborated with the aid of an example.
\end{abstract}

\section{Introduction}

Fractional calculus is the study of theory and applications of integrals and derivatives of an arbitrary (noninteger) order. This branch of mathematical analysis, extensively investigated in the recent years, has emerged as an effective and powerful tool for the mathematical modeling of several engineering and scientific phenomena. One of the key factors for the popularity of the subject is the nonlocal nature of fractional-order operators. Due to this reason, fractionalorder operators are used for describing the hereditary properties of many materials and processes. It clearly reflects from the related literature that the focus of investigation has shifted from classical integer-order models to fractionalorder models. For applications in applied and biomedical sciences and engineering, we refer the reader to the books [1-4]. For some recent work on the topic, see [5-25] and the references therein. The study of coupled systems of fractionalorder differential equations is quite important as such systems appear in a variety of problems of applied nature, especially in biosciences. For details and examples, the reader is referred to the papers [26-33] and the references cited therein.

Hybrid fractional differential equations have also been studied by several researchers. This class of equations involves the fractional derivative of an unknown function hybrid with the nonlinearity depending on it. Some recent results on hybrid differential equations can be found in a series of papers (see [34-37]).

Motivated by some recent studies on hybrid fractional differential equations, we consider the following Dirichlet boundary value problem of coupled hybrid fractional differential equations:

$$
\begin{gathered}
{ }^{c} D^{\delta}\left(\frac{x(t)}{f_{1}(t, x(t), y(t))}\right)=h_{1}(t, x(t), y(t)), \\
0<t<1, \quad 1<\delta \leq 2, \\
{ }^{c} D^{\omega}\left(\frac{y(t)}{f_{2}(t, x(t), y(t))}\right)=h_{2}(t, x(t), y(t)), \\
0<t<1, \quad 1<\omega \leq 2, \\
x(0)=x(1)=0, \quad y(0)=y(1)=0,
\end{gathered}
$$

where ${ }^{c} D^{\delta},{ }^{c} D^{\omega}$ denote the Caputo fractional derivative of orders $\delta, \omega$, respectively, $f_{i} \in C([0,1] \times \mathbb{R} \times \mathbb{R}, \mathbb{R} \backslash\{0\})$ and $h_{i} \in C([0,1] \times \mathbb{R} \times \mathbb{R}, \mathbb{R}), i=1,2$.

The aim of this paper is to obtain some existence results for the given problem. Our first theorem describes the uniqueness of solutions for the problem (1) by means of Banach's fixed point theorem. In the second theorem, we apply Leray-Schauder's alternative criterion to show the 
existence of solutions for the given problem. The paper is organized as follows. Section 2 contains some basic concepts and an auxiliary lemma, an important result for establishing our main results. In Section 3, we present the main results.

\section{Preliminaries}

In this section, some basic definitions on fractional calculus and an auxiliary lemma are presented $[1,2]$.

Definition 1. The Riemann-Liouville fractional integral of order $q$ for a continuous function $g$ is defined as

$$
I^{q} g(t)=\frac{1}{\Gamma(q)} \int_{0}^{t} \frac{g(s)}{(t-s)^{1-q}} d s, \quad q>0,
$$

provided that the integral exists.

Definition 2. For at least $n$-times continuously differentiable function $g:[0, \infty) \rightarrow \mathbb{R}$, the Caputo derivative of fractional-order $q$ is defined as

$$
\begin{array}{r}
{ }^{c} D^{q} g(t)=\frac{1}{\Gamma(n-q)} \int_{0}^{t}(t-s)^{n-q-1} g^{(n)}(s) d s, \\
n-1<q<n, \quad n=[q]+1,
\end{array}
$$

where $[q]$ denotes the integer part of the real number $q$.

Lemma 3 (auxiliary lemma). Given $\phi \in C([0,1], \mathbb{R})$, the integral solution of the problem

$$
\begin{gathered}
D^{\delta}\left(\frac{x(t)}{f(t, x(t), y(t))}\right)=\phi(t), \quad 0<t<1, \\
x(0)=x(1)=0
\end{gathered}
$$

is

$$
\begin{aligned}
& x(t)= f(t, x(t), y(t)) \\
& \times\left(\int_{0}^{t} \frac{(t-s)^{\delta-1}}{\Gamma(\delta)} \phi(s) d s-t \int_{0}^{1} \frac{(1-s)^{\delta-1}}{\Gamma(\delta)} \phi(s) d s\right), \\
& t \in[0,1]
\end{aligned}
$$

Proof. It is well known that the general solution of the fractional differential equation in (4) can be written as

$$
\frac{x(t)}{f(t, x(t), y(t))}=\int_{0}^{t} \frac{(t-s)^{\delta-1}}{\Gamma(\delta)} \phi(s) d s+c_{0} t+c_{1},
$$

where $c_{0}, c_{1} \in \mathbb{R}$ are arbitrary constants. Alternatively, we have

$$
\begin{aligned}
x(t)= & f(t, x(t), y(t)) \\
& \times\left(\int_{0}^{t} \frac{(t-s)^{\delta-1}}{\Gamma(\delta)} \phi(s) d s+c_{0} t+c_{1}\right), \quad t \in[0,1] .
\end{aligned}
$$

Using the given boundary conditions $x(0)=0=x(1)$ in (7), we find that

$$
c_{1}=0, \quad c_{0}=-\int_{0}^{1} \frac{(1-s)^{\delta-1}}{\Gamma(\delta)} \phi(s) d s .
$$

Substituting the values of $c_{0}, c_{1}$ in (7) yields the solution

$$
\begin{aligned}
& x(t)= f(t, x(t), y(t)) \\
& \times\left(\int_{0}^{t} \frac{(t-s)^{\delta-1}}{\Gamma(\delta)} \phi(s) d s-t \int_{0}^{1} \frac{(1-s)^{\delta-1}}{\Gamma(\delta)} \phi(s) d s\right), \\
& t \in[0,1] .
\end{aligned}
$$

This completes the proof.

\section{Main Results}

Let $W=\left\{w(t) \mid w(t) \in C^{1}([0,1])\right\}$ denote a Banach space equipped with the norm $\|w\|=\max \{|w(t)|, t \in[0,1]\}$, where $W=\mathscr{U}, \mathscr{V}$. Notice that the product space $(\mathcal{U} \times \mathscr{V},\|(x, y)\|)$ with the norm $\|(x, y)\|=\|x\|+\|y\|,(x, y) \in \mathcal{U} \times \mathscr{V}$ is also a Banach space.

In view of Lemma 3, we define an operator $\Theta: \mathscr{U} \times \mathscr{V} \rightarrow$ $\mathscr{U} \times \mathscr{V}$ by

$$
\Theta(x, y)(t)=\left(\begin{array}{l}
\Theta_{1}(x, y)(t) \\
\Theta_{2}(x, y)(t)
\end{array}\right)
$$

where

$$
\begin{aligned}
\Theta_{1}(x, y)(t)= & f_{1}(t, x(t), y(t)) \\
& \times\left(\int_{0}^{t} \frac{(t-s)^{\delta-1}}{\Gamma(\delta)} h_{1}(s, x(s), y(s)) d s\right. \\
& \left.\quad-t \int_{0}^{1} \frac{(1-s)^{\delta-1}}{\Gamma(\delta)} h_{1}(s, x(s), y(s)) d s\right), \\
\Theta_{2}(x, y)(t)= & f_{2}(t, x(t), y(t)) \\
& \times\left(\int_{0}^{t} \frac{(t-s)^{\omega-1}}{\Gamma(\omega)} h_{2}(s, x(s), y(s)) d s\right. \\
& \left.\quad-t \int_{0}^{1} \frac{(1-s)^{\omega-1}}{\Gamma(\omega)} h_{2}(s, x(s), y(s)) d s\right) .
\end{aligned}
$$

In the sequel, we need the following assumptions.

$\left(A_{1}\right)$ The functions $f_{i}(i=1,2)$ are continuous and bounded; that is, there exist positive numbers $\mu_{f_{i}}$ such that $\left|f_{i}(t, u, v)\right| \leq \mu_{f_{i}}, \forall(t, x, y) \in[0,1] \times \mathbb{R} \times \mathbb{R}$.

$\left(A_{2}\right)$ There exist real constants $\rho_{0}, \sigma_{0}>0$ and $\rho_{i}, \sigma_{i} \geq 0$ ( $i=$ $1,2)$ such that $\left|h_{1}(t, x, y)\right| \leq \rho_{0}+\rho_{1}|x|+\rho_{2}|y|$ and $\left|h_{2}(t, x, y)\right| \leq \sigma_{0}+\sigma_{1}|x|+\sigma_{2}|y|, \forall x, y \in \mathbb{R}, i=1,2$. 
For brevity, let us set

$$
\begin{aligned}
& v_{1}=\frac{2 \mu_{f_{1}}}{\Gamma(\delta+1)}, \quad \nu_{2}=\frac{2 \mu_{f_{2}}}{\Gamma(\omega+1)}, \\
& v_{0}=\min \left\{1-\left(\nu_{1} \rho_{1}+\nu_{2} \sigma_{1}\right), 1-\left(\nu_{1} \rho_{2}+\nu_{2} \sigma_{2}\right)\right\}, \\
& \rho_{i}, \sigma_{i} \geq 0 \quad(i=1,2) .
\end{aligned}
$$

Now we are in a position to present our first result that deals with the existence and uniqueness of solutions for the problem (1). This result is based on Banach's contraction mapping principle.

Theorem 4. Suppose that condition $\left(A_{1}\right)$ holds and that $h_{1}, h_{2}:[0,1] \times \mathbb{R}^{2} \rightarrow \mathbb{R}$ are continuous functions. In addition, there exist positive constants $\eta_{i}, \zeta_{i}, i=1,2$ such that

$$
\begin{array}{r}
\left|h_{1}\left(t, x_{1}, y_{1}\right)-h_{1}\left(t, x_{2}, y_{2}\right)\right| \leq \eta_{1}\left|x_{1}-x_{2}\right|+\eta_{2}\left|y_{1}-y_{2}\right|, \\
\left|h_{2}\left(t, x_{1}, y_{1}\right)-h_{2}\left(t, x_{2}, y_{2}\right)\right| \leq \zeta_{1}\left|x_{1}-x_{2}\right|+\zeta_{2}\left|y_{1}-y_{2}\right|, \\
\forall t \in[0,1], \quad x_{1}, x_{2}, y_{1}, y_{2} \in \mathbb{R} .
\end{array}
$$

If $\nu_{1}\left(\eta_{1}+\eta_{2}\right)+\nu_{2}\left(\zeta_{1}+\zeta_{2}\right)<1 \nu_{1}$ and $\nu_{2}$ are given by (12), then the problem (1) has a unique solution.

Proof. Let us set $\sup _{t \in[0,1]} h_{1}(t, 0,0)=\kappa_{1}<\infty, \sup _{t \in[0,1]} h_{2}(t$, $0,0)=\kappa_{2}<\infty$ and define a closed ball: $\bar{B}_{r}=\{(x, y) \in \mathscr{U} \times \mathscr{V}:$ $\|(x, y)\| \leq r\}$, where

$$
r \geq \frac{\kappa_{1} \nu_{1}+\kappa_{2} \nu_{2}}{1-\nu_{1}\left(\eta_{1}+\eta_{2}\right)-\nu_{2}\left(\zeta_{1}+\zeta_{2}\right)} .
$$

Then we show that $\Theta \bar{B}_{r} \subset \bar{B}_{r}$. For $(x, y) \in \bar{B}_{r}$, we obtain

$$
\begin{aligned}
& \left|\Theta_{1}(x, y)(t)\right| \\
& \begin{aligned}
\leq M_{f_{1}} \max _{t \in[0,1]}\left\{\int_{0}^{t} \frac{(t-s)^{\delta-1}}{\Gamma(\delta)}\left|h_{1}(s, x(s), y(s))\right| d s\right. \\
\left.+\int_{0}^{1} \frac{(1-s)^{\delta-1}}{\Gamma(\delta)}\left|h_{1}(s, x(s), y(s))\right| d s\right\}
\end{aligned} \\
& \begin{aligned}
\leq M_{f_{1}} \max _{t \in[0,1]}\left\{\int_{0}^{t} \frac{(t-s)^{\delta-1}}{\Gamma(\delta)}\right. \\
\quad \times\left(\left|h_{1}(s, x(s), y(s))-h_{1}(s, 0,0)\right|\right.
\end{aligned} \\
& \quad \times\left(\left|h_{1}(s, x(s), y(s))-h_{1}(s, 0,0)\right|\right. \\
& \left.\left.\quad+\left|h_{1}(s, 0,0)\right|\right) d s\right\} \\
& \begin{array}{c}
\leq \frac{2 M_{f_{1}}}{\Gamma(\delta+1)}\left(\eta_{1}\|x\|+\eta_{2}\|y\|+\kappa_{1}\right) \\
\leq v_{1}\left[\left(\eta_{1}+\eta_{2}\right) r+\kappa_{1}\right] .
\end{array}
\end{aligned}
$$

Hence

$$
\left\|\Theta_{1}(x, y)\right\| \leq v_{1}\left[\left(\eta_{1}+\eta_{2}\right) r+\kappa_{1}\right]
$$

Working in a similar manner, one can find that

$$
\left\|\Theta_{2}(x, y)\right\| \leq v_{2}\left[\left(\zeta_{1}+\zeta_{2}\right) r+\kappa_{2}\right]
$$

From (17) and (18), it follows that $\|\Theta(x, y)\| \leq r$.

Next, for $\left(x_{1}, y_{1}\right),\left(x_{2}, y_{2}\right) \in \mathscr{U} \times \mathscr{V}$ and for any $t \in[0,1]$, we have

$$
\begin{aligned}
& \left|\Theta_{1}\left(x_{2}, y_{2}\right)(t)-\Theta_{1}\left(x_{1}, y_{1}\right)(t)\right| \\
& \leq \mu_{f_{1}} \int_{0}^{t} \frac{(t-s)^{\delta-1}}{\Gamma(\delta)} \mid h_{1}\left(s, x_{2}(s), y_{2}(s)\right) \\
& \quad-f\left(s, x_{1}(s), y_{1}(s)\right) \mid d s \\
& \quad+\int_{0}^{1} \frac{(1-s)^{\delta-1}}{\Gamma(\delta)} \mid h_{1}\left(s, x_{2}(s), y_{2}(s)\right) \\
& \quad-f\left(s, x_{1}(s), y_{1}(s)\right) \mid d s \\
& \leq \frac{2 \mu_{f_{1}}}{\Gamma(\delta+1)}\left(m_{1}\left|u_{2}-u_{1}\right|+m_{2}\left|v_{2}-v_{1}\right|\right) \\
& \leq v_{1}\left(\eta_{1}\left\|x_{2}-x_{1}\right\|+\eta_{2}\left\|y_{2}-y_{1}\right\|\right) \\
& \leq v_{1}\left(m_{1}+m_{2}\right)\left(\left\|x_{2}-x_{1}\right\|+\left\|y_{2}-y_{1}\right\|\right),
\end{aligned}
$$

which yields

$$
\begin{aligned}
& \left\|\Theta_{1}\left(x_{2}, y_{2}\right)(t)-\Theta_{1}\left(x_{1}, y_{1}\right)\right\| \\
& \quad \leq v_{1}\left(m_{1}+m_{2}\right)\left(\left\|x_{2}-x_{1}\right\|+\left\|y_{2}-y_{1}\right\|\right) .
\end{aligned}
$$

Similarly, one can get

$$
\begin{aligned}
& \left\|\Theta_{2}\left(x_{2}, y_{2}\right)(t)-\Theta_{2}\left(x_{1}, y_{1}\right)\right\| \\
& \quad \leq v_{2}\left(\zeta_{1}+\zeta_{2}\right)\left(\left\|x_{2}-x_{1}\right\|+\left\|y_{2}-y_{1}\right\|\right) .
\end{aligned}
$$

From (20) and (21), we deduce that

$$
\begin{aligned}
& \left\|\Theta\left(x_{2}, y_{2}\right)-\Theta\left(x_{1}, y_{1}\right)\right\| \leq\left[v_{1}\left(\eta_{1}+\eta_{2}\right)+v_{2}\left(\zeta_{1}+\zeta_{2}\right)\right] \\
& \quad \times\left(\left\|x_{2}-x_{1}\right\|+\left\|y_{2}-y_{1}\right\|\right) .
\end{aligned}
$$

In view of condition $\nu_{1}\left(\eta_{1}+\eta_{2}\right)+\nu_{2}\left(\zeta_{1}+\zeta_{2}\right)<1$, it follows that $\Theta$ is a contraction. So Banach's fixed point theorem applies and hence the operator $\Theta$ has a unique fixed point. This, in turn, implies that the problem (1) has a unique solution on $[0,1]$. This completes the proof. 
Example 5. Consider the following coupled system of hybrid fractional differential equations:

$$
\begin{gathered}
{ }^{c} D^{3 / 2}\left(\frac{u(t)}{(1 / 2)(|\sin u(t)|+1)}\right)=\frac{1}{4(t+2)^{2}} \frac{|u(t)|}{1+|u(t)|}+1 \\
+\frac{1}{32} \sin ^{2} v(t), \quad t \in[0,1], \\
{ }^{c} D^{3 / 2}\left(\frac{v(t)}{(1 / 2)(|\cos u(t)|+1)}\right)=\frac{1}{32 \pi} \sin (2 \pi u(t)) \\
+\frac{|v(t)|}{16(1+|v(t)|)}+\frac{1}{2}, \quad t \in[0,1], \\
u(0)=0, \quad u(1)=0, \\
v(0)=0, \quad v(1)=0 .
\end{gathered}
$$

Here $\delta=3 / 2, \omega=3 / 2, f_{1}(t, u, v)=(1 / 2)(|\sin u(t)|+1), f_{2}(t$, $u, v)=(1 / 2)(|\cos u(t)|+1), h_{1}(t, x, y)=\left(1 / 4(t+2)^{2}\right)(|x| /(1+$ $|x|))+1+(1 / 32) \sin ^{2} y$, and $h_{2}(t, x, y)=(1 / 32 \pi) \sin (2 \pi x)+$ $|y| / 16(1+|y|)+1 / 2$. Note that

$$
\begin{gathered}
\left|h_{1}\left(t, x_{2}, y_{2}\right)-h_{1}\left(t, x_{1}, y_{1}\right)\right| \leq \frac{1}{16}\left|x_{2}-x_{1}\right|+\frac{1}{16}\left|y_{2}-y_{1}\right| \\
\left|h_{2}\left(t, x_{2}, y_{2}\right)-h_{2}\left(t, x_{1}, y_{1}\right)\right| \leq \frac{1}{16}\left|x_{2}-x_{1}\right|+\frac{1}{16}\left|y_{2}-y_{1}\right|, \\
v_{1}\left(\eta_{1}+\eta_{2}\right)+v_{2}\left(\zeta_{1}+\zeta_{2}\right)=\frac{2}{3 \sqrt{\pi}} \approx 0.3762217<1 .
\end{gathered}
$$

Thus all the conditions of Theorem 4 are satisfied and, consequently, there exists a unique solution for the problem (23) on $[0,1]$.

In our second result, we discuss the existence of solutions for the problem (1) by means of Leray-Schauder alternative.

Lemma 6 (Leray-Schauder alternative [38, page 4]). Let $\mathscr{F}$ : $\mathscr{G} \rightarrow \mathscr{G}$ be a completely continuous operator (i.e., a map that is restricted to any bounded set in $\mathscr{G}$ is compact). Let $\mathscr{P}(\mathscr{F})=$ $\{x \in \mathscr{G}: x=\lambda \mathscr{F} x$ for some $0<\lambda<1\}$. Then either the set $\mathscr{P}(\mathscr{F})$ is unbounded or $\mathscr{F}$ has at least one fixed point.

Theorem 7. Assume that conditions $\left(A_{1}\right)$ and $\left(A_{2}\right)$ hold. Furthermore, it is assumed that $\nu_{1} \rho_{1}+\nu_{2} \sigma_{1}<1$ and $\nu_{1} \rho_{2}+$ $\nu_{2} \sigma_{2}<1$, where $\nu_{1}$ and $\nu_{2}$ are given by (12). Then the boundary value problem (1) has at least one solution.

Proof. We will show that the operator $\Theta: \mathscr{U} \times \mathscr{V} \rightarrow \mathscr{U} \times \mathscr{V}$ satisfies all the assumptions of Lemma 6. In the first step, we prove that the operator $\Theta$ is completely continuous. Clearly, it follows by the continuity of functions $f_{1}, f_{2}, h_{1}$, and $h_{2}$ that the operator $\Theta$ is continuous.
Let $\mathscr{M} \subset \mathscr{U} \times \mathscr{V}$ be bounded. Then we can find positive constants $N_{1}$ and $N_{2}$ such that

$$
\begin{array}{r}
\left|h_{1}(t, x(t), y(t))\right| \leq N_{1}, \quad\left|h_{2}(t, x(t), y(t))\right| \leq N_{2}, \\
\forall(x, y) \in \mathscr{M} .
\end{array}
$$

Thus for any $x, y \in \mathscr{M}$, we can get

$$
\begin{aligned}
& \left|\Theta_{1}(x, y)(t)\right| \\
& \quad \leq \mu_{f_{1}}\left\{\int_{0}^{t} \frac{(t-s)^{\delta-1}}{\Gamma(\delta)}\left|h_{1}(s, x(s), y(s))\right| d s\right. \\
& \left.\quad+\int_{0}^{1} \frac{(1-s)^{\delta-1}}{\Gamma(\delta)}\left|h_{1}(s, x(s), y(s))\right| d s\right\} \\
& \leq \mu_{f_{1}} N_{1} \frac{2}{\Gamma(\delta+1)},
\end{aligned}
$$

which yields

$$
\left\|\Theta_{1}(x, y)\right\| \leq \frac{2 \mu_{f_{1}} N_{1}}{\Gamma(\delta+1)}=N_{1} \nu_{1} .
$$

In a similar manner, one can show that

$$
\left\|\Theta_{2}(x, y)\right\| \leq \frac{2 \mu_{f_{2}} N_{2}}{\Gamma(\omega+1)}=N_{2} \nu_{2} .
$$

From the inequalities (27) and (28), we deduce that the operator $\Theta$ is uniformly bounded.

Now we show that the operator $\Theta$ is equicontinuous. For that, we take $\tau_{1}, \tau_{2} \in[0,1]$ with $\tau_{1}<\tau_{2}$ and obtain

$$
\begin{aligned}
& \left|\Theta_{1}\left(x\left(\tau_{2}\right), y\left(\tau_{2}\right)\right)-\Theta_{1}\left(x\left(\tau_{1}\right), y\left(\tau_{1}\right)\right)\right| \\
& \leq \mu_{f_{1}} N_{1}\left|\int_{0}^{\tau_{1}} \frac{\left(\tau_{1}-s\right)^{\delta-1}}{\Gamma(\delta)} d s-\int_{0}^{\tau_{2}} \frac{\left(\tau_{2}-s\right)^{\delta-1}}{\Gamma(\delta)} d s\right| \\
& +\mu_{f_{1}} N_{1}\left|\tau_{2}-\tau_{1}\right| \int_{0}^{1} \frac{(1-s)^{\delta-1}}{\Gamma(\delta)} d s \\
& =\mu_{f_{1}} N_{1} \mid \int_{0}^{\tau_{1}} \frac{\left(\tau_{1}-s\right)^{\delta-1}-\left(\tau_{2}-s\right)^{\delta-1}}{\Gamma(\delta)} d s \\
& \quad+\mu_{f_{1}} N_{1}\left|\tau_{2}-\tau_{1}\right| \int_{0}^{1} \frac{(1-s)^{\delta-1}}{\Gamma(\delta)} d s \longrightarrow 0, \\
& \quad+\mu_{f_{2}} N_{2}\left|\tau_{2}-\tau_{1}\right| \int_{0}^{1} \frac{(1-s)^{\omega-1}}{\Gamma(\omega)} d s, \\
& \left|\Theta_{2}\left(x\left(\tau_{2}\right), y\left(\tau_{2}\right)\right)-\Theta_{2}\left(x\left(\tau_{1}\right), y\left(\tau_{1}\right)\right)\right| \\
& \leq \mu_{f_{2}} N_{2} \mid \int_{0}^{\tau_{1}} \frac{\left(\tau_{1}-s\right)^{\omega-1}-\left(\tau_{2}-s\right)^{\omega-1}}{\Gamma(\omega)} d s \\
& \Gamma(\omega) d s \mid \\
& \tau_{2} \frac{\left(\tau_{2}-s\right)^{\omega-1} \mid}{\Gamma(\delta)} d s \mid
\end{aligned}
$$


which tend to 0 independently of $(x, y)$. This implies that the operator $\Theta(x, y)$ is equicontinuous. Thus, by the above findings, the operator $\Theta(x, y)$ is completely continuous.

In the next step, it will be established that the set $\mathscr{P}=$ $\{(x, y) \in \mathscr{U} \times \mathscr{V} \mid(x, y)=\lambda \Theta(x, y), 0 \leq \lambda \leq 1\}$ is bounded. Let $(x, y) \in \mathscr{P}$; then we have $(x, y)=\lambda \Theta(x, y)$. Thus, for any $t \in[0,1]$, we can write

$$
x(t)=\lambda \Theta_{1}(x, y)(t), \quad y(t)=\lambda \Theta_{2}(x, y)(t) .
$$

Then

$$
\begin{aligned}
& |x(t)| \leq \frac{2 \mu_{f_{1}}}{\Gamma(\delta+1)}\left(\rho_{0}+\rho_{1}\|x\|+\rho_{2}\|y\|\right), \\
& |y(t)| \leq \frac{2 \mu_{f_{2}}}{\Gamma(\omega+1)}\left(\sigma_{0}+\sigma_{1}\|x\|+\sigma_{2}\|y\|\right),
\end{aligned}
$$

which imply that

$$
\begin{gathered}
\|x\| \leq \nu_{1}\left(\rho_{0}+\rho_{1}\|x\|+\rho_{2}\|y\|\right), \\
\|y\| \leq v_{2}\left(\sigma_{0}+\sigma_{1}\|x\|+\sigma_{2}\|y\|\right) .
\end{gathered}
$$

In consequence, we have

$$
\begin{aligned}
\|x\| & +\|y\|=\left(\nu_{1} \rho_{0}+v_{2} \sigma_{0}\right) \\
& +\left(\nu_{1} \rho_{1}+v_{2} \sigma_{1}\right)\|x\|+\left(v_{1} \rho_{2}+v_{2} \sigma_{2}\right)\|y\|,
\end{aligned}
$$

which, in view of (13), can be expressed as

$$
\|(x, y)\| \leq \frac{v_{1} \rho_{0}+\nu_{2} \sigma_{0}}{v_{0}} .
$$

This shows that the set $\mathscr{P}$ is bounded. Hence all the conditions of Lemma 6 are satisfied and consequently the operator $\Theta$ has at least one fixed point, which corresponds to a solution of the problem (1). This completes the proof.

\section{Conflict of Interests}

The authors declare that there is no conflict of interests regarding the publication of this paper.

\section{Acknowledgment}

This paper was supported by the Deanship of Scientific Research (DSR), King Abdulaziz University, Jeddah, Saudi Arabia. The authors, therefore, acknowledge technical and financial support of KAU.

\section{References}

[1] I. Podlubny, Fractional Differential Equations, Academic Press, San Diego, Calif, USA, 1999.

[2] A. A. Kilbas, H. M. Srivastava, and J. J. Trujillo, Theory and Applications of Fractional Differential Equations, vol. 204 of North-Holland Mathematics Studies, Elsevier Science, Amsterdam, The Netherlands, 2006.
[3] V. Lakshmikantham, S. Leela, and J. Vasundhara Devi, Theory of Fractional Dynamic Systems, Cambridge Academic Publishers, Cambridge, Mass, USA, 2009.

[4] J. Sabatier, O. P. Agrawal, and J. A. T. Machado, Eds., Advances in Fractional Calculus: Theoretical Developments and Applications in Physics and Engineering, Springer, Dordrecht, The Netherlands, 2007.

[5] B. Ahmad, J. J. Nieto, and J. Pimentel, "Some boundary value problems of fractional differential equations and inclusions," Computers \& Mathematics with Applications, vol. 62, no. 3, pp. 1238-1250, 2011.

[6] B. Ahmad, S. K. Ntouyas, and A. Alsaedi, "New existence results for nonlinear fractional differential equations with threepoint integral boundary conditions," Advances in Difference Equations, vol. 2011, Article ID 107384, 11 pages, 2011.

[7] B. Ahmad and S. K. Ntouyas, "A four-point nonlocal integral boundary value problem for fractional differential equations of arbitrary order," Electronic Journal of Qualitative Theory of Differential Equations, no. 22, 15 pages, 2011.

[8] J. R. Graef, L. Kong, and Q. Kong, "Application of the mixed monotone operator method to fractional boundary value problems," Fractional Differential Calculus, vol. 2, no. 1, pp. 554-567, 2011.

[9] D. Băleanu, R. P. Agarwal, O. G. Mustafa, and M. Coşulschi, "Asymptotic integration of some nonlinear differential equations with fractional time derivative," Journal of Physics A: Mathematical and Theoretical, vol. 44, no. 5, Article ID 055203, 9 pages, 2011.

[10] F. T. Akyildiz, H. Bellout, K. Vajravelu, and R. A. Van Gorder, "Existence results for third order nonlinear boundary value problems arising in nano boundary layer fluid flows over stretching surfaces," Nonlinear Analysis: Real World Applications, vol. 12, no. 6, pp. 2919-2930, 2011.

[11] R. Sakthivel, N. I. Mahmudov, and J. J. Nieto, "Controllability for a class of fractional-order neutral evolution control systems," Applied Mathematics and Computation, vol. 218, no. 20, pp. 10334-10340, 2012.

[12] R. P. Agarwal, D. O’ Regan, and S. Staněk, "Positive solutions for mixed problems of singular fractional differential equations," Mathematische Nachrichten, vol. 285, no. 1, pp. 27-41, 2012.

[13] J. Wang, Y. Zhou, and M. Medved, "Qualitative analysis for nonlinear fractional differential equations via topological degree method," Topological Methods in Nonlinear Analysis, vol. 40, no. 2, pp. 245-271, 2012.

[14] L. Zhang, B. Ahmad, G. Wang, and R. P. Agarwal, "Nonlinear fractional integro-differential equations on unbounded domains in a Banach space," Journal of Computational and Applied Mathematics, vol. 249, pp. 51-56, 2013.

[15] W. Zhou, Y. Chu, and D. Baleanu, "Uniqueness and existence of positive solutions for a multi-point boundary value problem of singular fractional differential equations," Advances in Difference Equations, vol. 2013, article 114, 2013.

[16] B. Ahmad, S. K. Ntouyas, and A. Alsaedi, "A study of nonlinear fractional differential equations of arbitrary order with Riemann-Liouville type multistrip boundary conditions," Mathematical Problems in Engineering, vol. 2013, Article ID 320415, 9 pages, 2013.

[17] J. R. Graef, L. Kong, and M. Wang, "Existence and uniqueness of solutions for a fractional boundary value problem on a graph," Fractional Calculus and Applied Analysis, vol. 17, no. 2, pp. 499510, 2014. 
[18] F. Punzo and G. Terrone, "On the Cauchy problem for a general fractional porous medium equation with variable density," Nonlinear Analysis: Theory, Methods \& Applications, vol. 98, pp. 27-47, 2014.

[19] X. Liu, Z. Liu, and X. Fu, "Relaxation in nonconvex optimal control problems described by fractional differential equations," Journal of Mathematical Analysis and Applications, vol. 409, no. 1, pp. 446-458, 2014.

[20] K. Razminia, A. Razminia, and J. A. Tenreiro Machado, "Analysis of diffusion process in fractured reservoirs using fractional derivative approach," Communications in Nonlinear Science and Numerical Simulation, vol. 19, no. 9, pp. 3161-3170, 2014.

[21] J. Choi and P. Agarwal, "Certain fractional integral inequalities involving hypergeometric operators," East Asian Mathematical Journal, vol. 30, pp. 283-291, 2014.

[22] J. Choi and P. Agarwal, "Certain new pathway type fractional integral inequalities," Honam Mathematical Journal, vol. 36, no. 2, pp. 437-447, 2014.

[23] D. Baleanu and P. Agarwal, "Certain inequalities involving the fractional q-integral operators," Abstract and Applied Analysis, vol. 2014, Article ID 371274, 10 pages, 2014.

[24] J. Choi and P. Agarwal, "Some new Saigo type fractional integral inequalities and their $q$-analogues," Abstract and Applied Analysis, vol. 2014, Article ID 579260, 11 pages, 2014.

[25] P. Agarwal, "Further results on fractional calculus of Saigo operators," Applications and Applied Mathematics, vol. 7, no. 2, pp. 585-594, 2012.

[26] B. Ahmad and J. J. Nieto, "Existence results for a coupled system of nonlinear fractional differential equations with threepoint boundary conditions," Computers \& Mathematics with Applications, vol. 58, no. 9, pp. 1838-1843, 2009.

[27] X. Su, "Boundary value problem for a coupled system of nonlinear fractional differential equations," Applied Mathematics Letters, vol. 22, no. 1, pp. 64-69, 2009.

[28] J. Wang, H. Xiang, and Z. Liu, "Positive solution to nonzero boundary values problem for a coupled system of nonlinear fractional differential equations," International Journal of Differential Equations, vol. 2010, Article ID 186928, 12 pages, 2010.

[29] J. Sun, Y. Liu, and G. Liu, "Existence of solutions for fractional differential systems with antiperiodic boundary conditions," Computers \& Mathematics with Applications, vol. 64, no. 6, pp. 1557-1566, 2012.

[30] S. K. Ntouyas and M. Obaid, "A coupled system of fractional differential equations with nonlocal integral boundary conditions," Advances in Difference Equations, vol. 2012, article 130, 2012.

[31] Z. Hu and W. Liu, "Solvability of a coupled system of fractional differential equations with periodic boundary conditions at resonance," Ukrainian Mathematical Journal, vol. 65, no. 11, pp. 1619-1633, 2014.

[32] L. Cheng, W. Liu, and Q. Ye, "Boundary value problem for a coupled system of fractional differential equations with $p$ Laplacian operator at resonance," Electronic Journal of Differential Equations, no. 60, 12 pages, 2014.

[33] B. Ahmad and S. K. Ntouyas, "A fully Hadamard type integral boundary value problem of a coupled system of fractional differential equations," Fractional Calculus and Applied Analysis, vol. 17, no. 2, pp. 348-360, 2014.

[34] Y. Zhao, S. Sun, Z. Han, and Q. Li, "Theory of fractional hybrid differential equations," Computers \& Mathematics with Applications, vol. 62, no. 3, pp. 1312-1324, 2011.
[35] S. Sun, Y. Zhao, Z. Han, and Y. Li, “The existence of solutions for boundary value problem of fractional hybrid differential equations," Communications in Nonlinear Science and Numerical Simulation, vol. 17, no. 12, pp. 4961-4967, 2012.

[36] B. Ahmad and S. K. Ntouyas, "An existence theorem for fractional hybrid differential inclusions of HADamard type with DIRichlet boundary conditions," Abstract and Applied Analysis, vol. 2014, Article ID 705809, 7 pages, 2014.

[37] B. C. Dhage and S. K. Ntouyas, "Existence results for boundary value problems for fractional hybrid differential inclucions," Topological Methods in Nonlinear Analysis, In press.

[38] A. Granas and J. Dugundji, Fixed Point Theory, Springer, New York, NY, USA, 2003. 


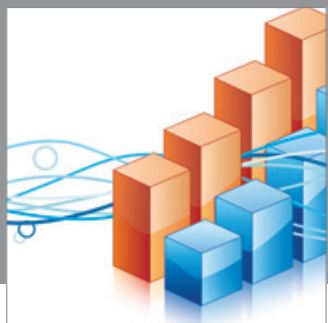

Advances in

Operations Research

mansans

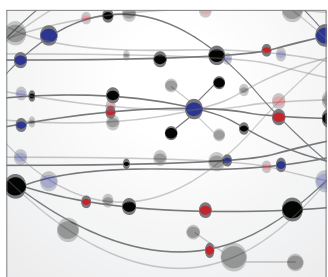

The Scientific World Journal
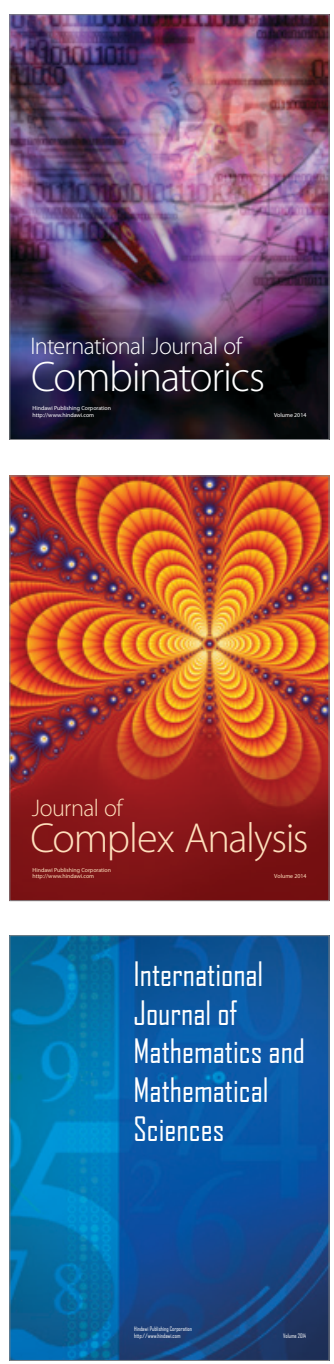
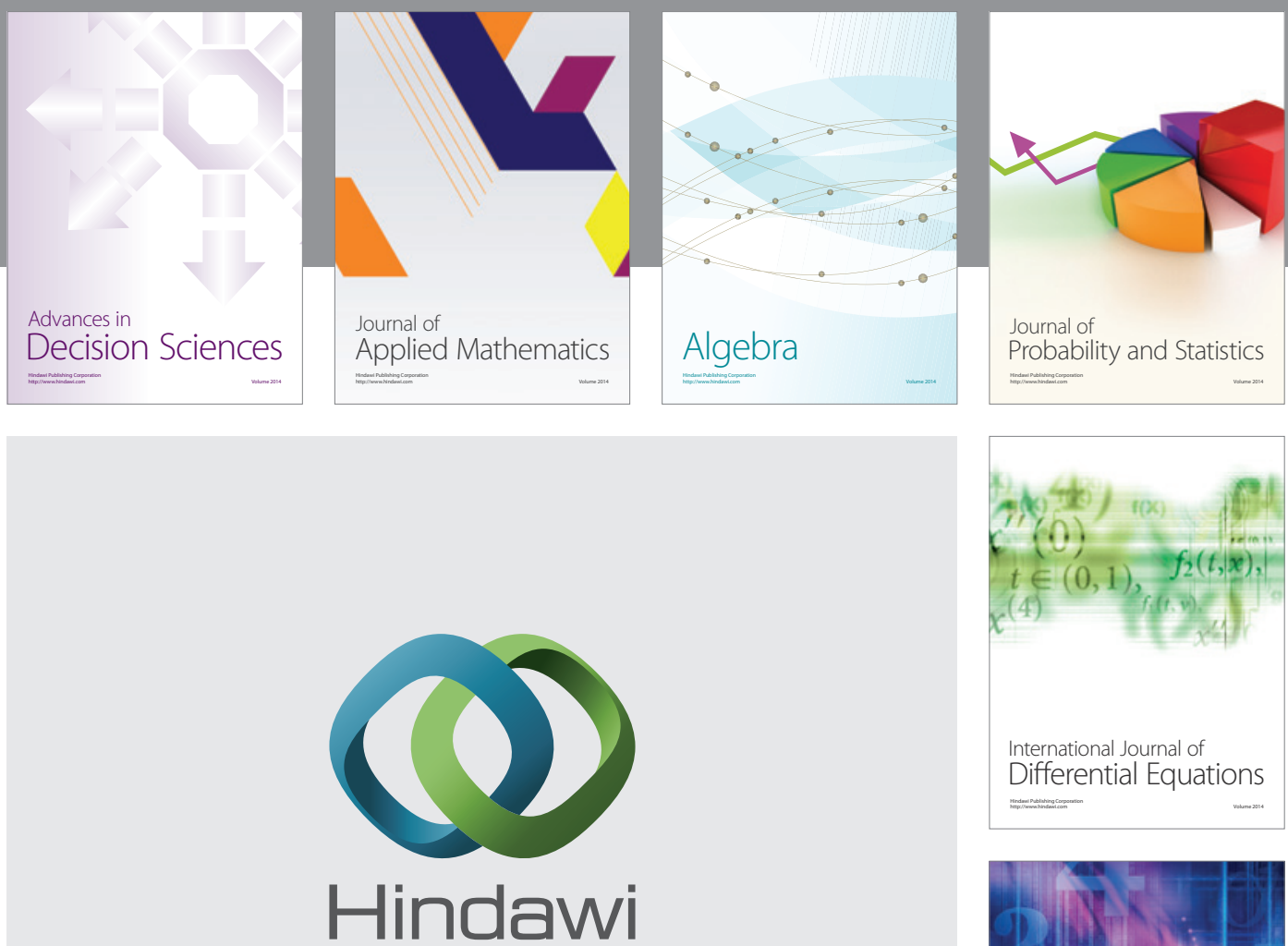

Submit your manuscripts at http://www.hindawi.com
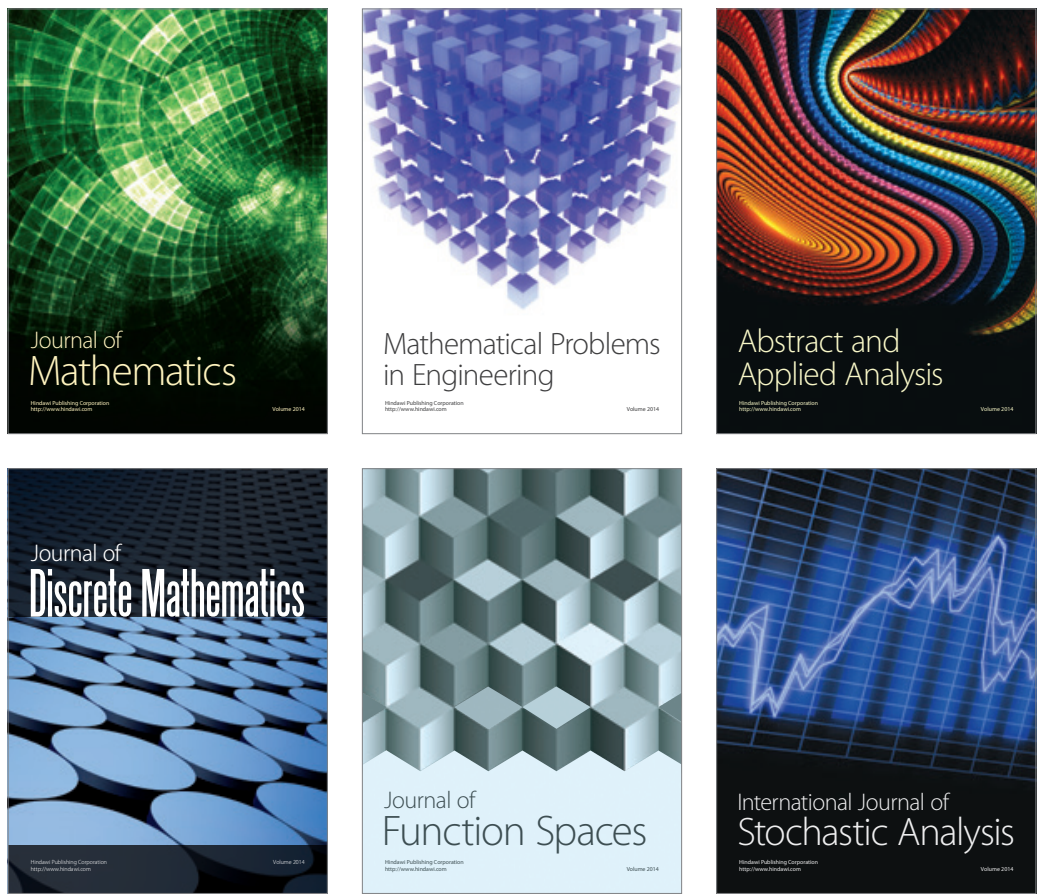

Journal of

Function Spaces

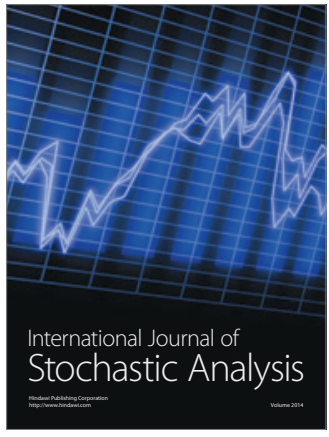

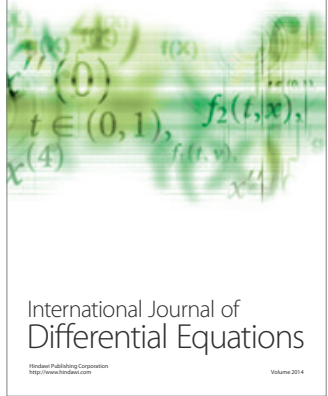
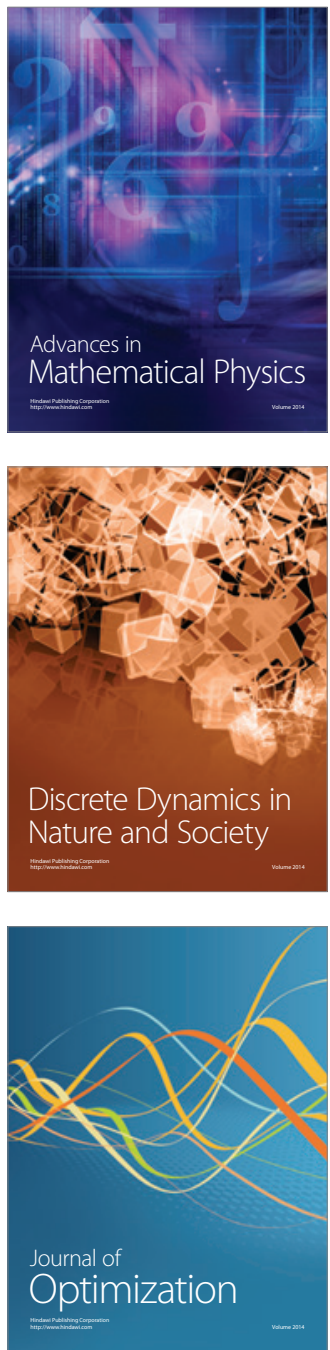